13

\title{
Ионно-пучковые и рентгеновские методы элементной диагностики тонкопленочных покрытий
}

\author{
(C) B.K. Егоров ${ }^{1}$, E.B. Егоров ${ }^{1,2,3}$, М.C. Асранасьев ${ }^{2}$ \\ ${ }^{1}$ Институт проблем технологии микроэлектроники РАН, \\ Черноголовка, Россия \\ ${ }^{2}$ Институт радиотехники и электроники РАН, \\ Фрязино, Россия \\ ${ }^{3}$ Финансовый университет при правительстве РФ, \\ Москва, Россия \\ E-mail: egorov@iptm.ru
}

Поступила в Редакцию 16 июля 2019 г.

В окончательной редакции 16 июля 2019 г.

Принята к публикации 25 июля 2019 г.

\begin{abstract}
Показано, как совместное использование методов резерфордовского обратного рассеяния ионов, рентгенофлуоресцентного анализа в условиях полного внешнего отражении потока возбуждающего жесткого рентгеновского излучения и регистрации выхода рентгеновского излучения при ионном возбуждении позволяет эффективно диагностировать элементный состав тонкопленочных покрытий и пленок сухих остатков жидкостей. Представлена краткая характеристика этих методов и особенностей их экспериментального применения. Приведены примеры комплексного методического анализа реальных объектов. Указано на возможность повышения эффективности методов рентгенофлуоресцентного анализа материалов за счет включения в рентгенооптические схемы экспериментальных измерений плоских рентгеновских волноводоврезонаторов.
\end{abstract}

Ключевые слова: резерфордовское обратное рассеяние, рентгенофлуоресцентная диагностика, рентгенофлуоресцентный анализ, тонкопленочные покрытия, пленки сухих остатков жидкостей.

DOI: $10.21883 /$ FTT.2019.12.48608.47ks

\section{1. Введение}

Задача эффективной элементной диагностики материалов, наряду с их структурным и фазовым анализом, является определяющей в условиях поиска новых материальных структур с необходимыми свойствами. Эта задача становится особенно актуальной при разработке защитных и функциональных покрытий, а также при выполнении модификации поверхностных слоев материалов имплантационными и диффузионными методами, причем в этих случаях зоны аналитического интереса оказываются наноразмерными. Кроме того, постановка технологического процесса часто требует от применяемых аналитических методик минимальной деструкции диагностируемого объекта, экспресности измерений и обеспечения их количественности. При этом последнее требование, в плане методического выбора, оказывается особенно важным, ввиду отсутствия надежных эталонов при диагностике наноразмерных объектов.

Хорошо известно, что единственным инструментальным методом (за исключением взвешивания), не требующим использования эталонов и стандартов, является резерфордовское обратное рассеяние (РОР) ионов [1]. Этот метод на базе первых принципов позволяет с точностью до $1 \%$ аппроксимировать полученные спектры. В то же время, метод характеризуется достаточно низкой аналитической чувствительностью. При его использовании пределы обнаружения примесных компонентов оказывается не лучше $0.1 \%$ at. Поэтому измерения с помощью РОР спектрометрии полезны для количественной диагностики элементного содержания структурообразующих компонентов, а также при необходимости определения элементных концентрационных профилей по толщине пленочных покрытий и поверхностных слоев объемных структур. Для диагностики присутствия в них следовых концентраций модифицирующих или случайных примесей эффективно применение специальных методов рентгенофлуоресцентного анализа, реализуемых при ионно-пучковом возбуждении выхода характеристической рентгенофлуоресценции (PIXE) [2], а также при регистрации этого выхода, возбуждаемого потоком жесткого рентгеновского излучения в условиях его полного внешнего отражения на поверхности изучаемого объекта (РФА ПВО) [3]. Приведенные ниже экспериментальные данные показывают, что совместное применение этих трех диагностических методов позволяют исчерпывающе количественно контролировать элементный состав тонкопленочных покрытий и тонких приповерхностных слоев материалов.

\section{2. Диагностические возможности РФА ПВО-спектрометрии}

Рентгенофлуоресцентный анализ элементного состава материалов является наиболее распространенным мето- 

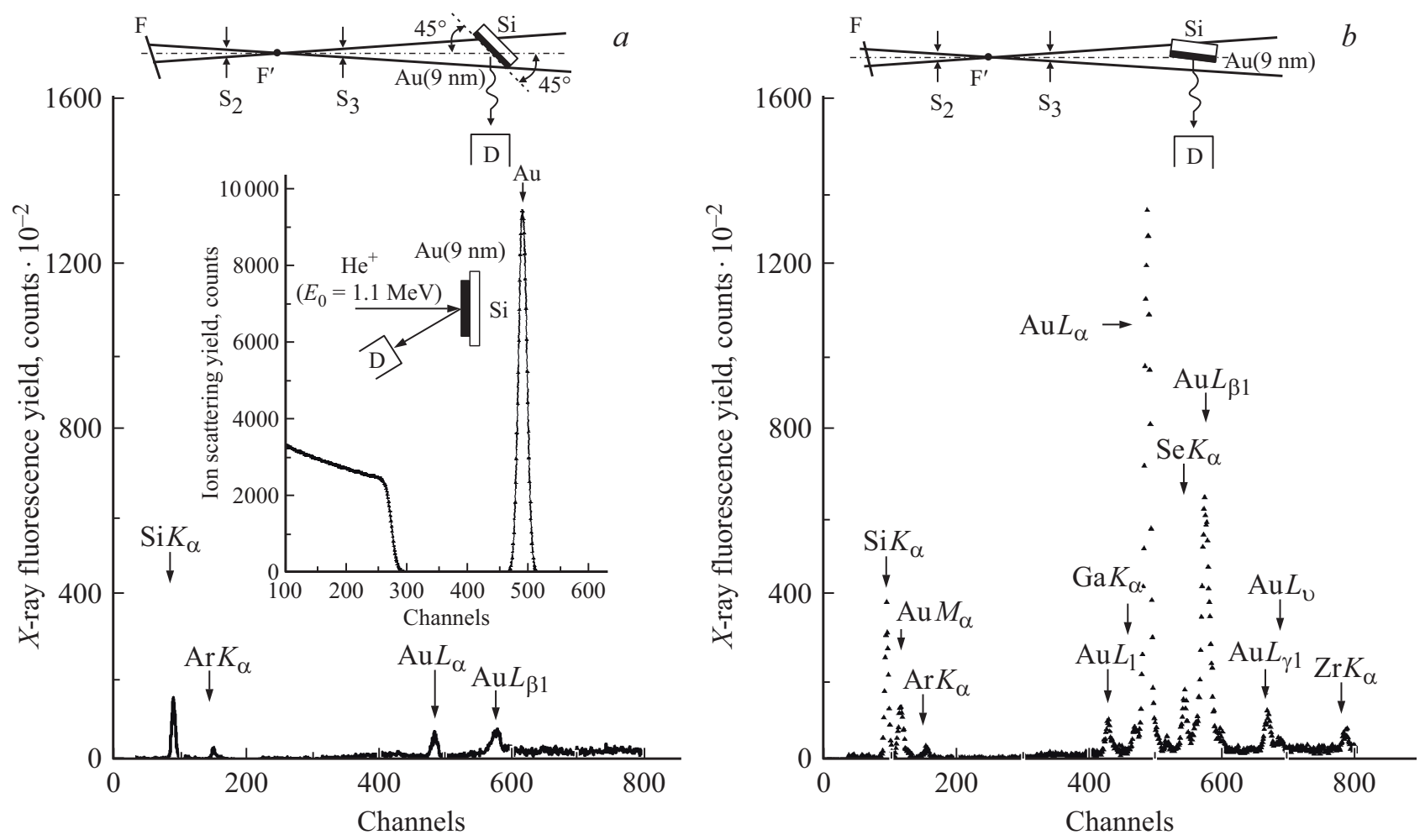

Рис. 1. Спектры рентгеновской флуоресценции образца $\mathrm{Au}(9 \mathrm{~nm}) / \mathrm{Si}$, полученные в геометрии РФА ПВО-измерений $(a)$ и в условиях стандартной РФА диагностики $(b)$, зарегистрированные в идентичных условиях функционирования источника излучения БСВ-27 (Мо) и формирования потока возбуждения. Энергетическая цена канала $20 \mathrm{eV} / \mathrm{channel}$. На врезке показан спектр РОР исследованного образца.

дом подобной диагностики. В то же время стандартный подход к применению этого метода как в условиях рентгеновского [4], так и электронного [5] возбуждения выхода характеристической рентгенофлуоресценции, характеризующийся углом падения потока возбуждения к поверхности изучаемого объекта $\theta=45^{\circ}$ и отбором анализируемого потока, соответствующим такому же углу (рис. $1, a$ ), имеет ряд аналитических недостатков. Важнейшим из них является наличие „матричного эффекта“, связанного с взаимным влиянием возбуждаемых в объекте рентгенофлуоресцентных характеристических излучений [6]. Кроме того, вследствие достаточно большой толщины возбуждаемого в объекте исследований материального слоя $(5-10 \mu \mathrm{m})$, стандартный подход характеризуется заметной величиной фоновой составляющей, являющейся критическим параметром при определении пределов обнаружения примесных компонентов [7]. Поэтому применение рентгенофлуоресцентного анализа в стандартной экспериментальной геометрии представляется малоэффективным для элементной диагностики тонкопленочных покрытий и приповерхностных слоев материалов. Поиск условий повышения эффективности такой диагностики привел к появлению РФА ПВО-спектрометрии - выполнению рентгенофлуоресцентного анализа в условиях полного внешнего отражения потока возбуждения [8]. В этих условиях толщина возбуждаемого поверхностного слоя изучаемого объекта уменьшается до значений $3-5 \mathrm{~nm}$, что, в свою очередь, исключает влияние на регистрируемый спектр матричного эффекта и позволяет снизить величину фонового вклада. В итоге, пределы обнаружения метода снижаются до 1 ppm. Кроме того, возбуждение поверхностного слоя изучаемого объекта в условиях РФА ПВО измерений оказывается более эффективным. В результате интенсивность выхода характеристических линий в сравнении со стандартной геометрией РФА измерений резко возрастает. Это хорошо иллюстрирует рис. 1, представляющий спектры РФА и РФА ПВО, полученные для тонкопленочного золотого покрытия на кремнии. Там же приведен спектр РОР этого покрытия.

Поскольку в РФА ПВО-спектрометрии возбуждается тонкий приповерхностный слой изучаемого объекта, критическим параметром методики является значение радиационной плотности потока возбуждения. Увеличение эффективности РФА ПВО-измерений путем роста этого параметра может быть достигнуто либо за счет примитивного увеличения мощности источника излучения, либо путем поиска оригинальных решений концентрации радиационного потока. Подобное решение было найдено в рамках разработки специфического устройства рентгеновской нанофотоники - плоского рентгеновского волновода-резонатора (ПРВР) [9]. Это устройство позволяет формировать нитевидные потоки рентгеновского излучения наноразмерной ширины с повышением радиационной плотности формируемого 

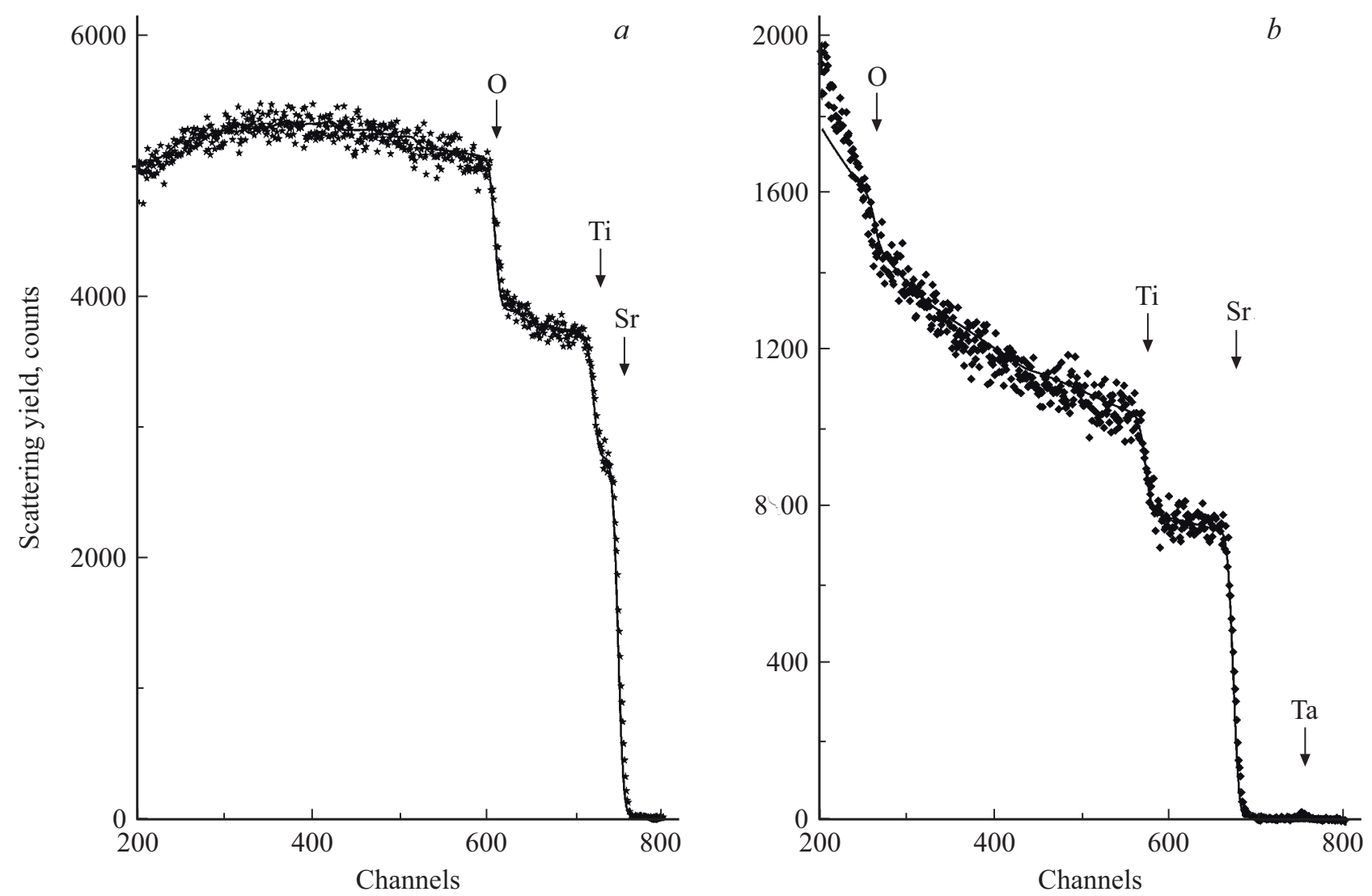

Рис. 2. Спектры РОР потоков ионов $\mathrm{He}^{+}(a)$ и $\mathrm{H}^{+}(b)$, зарегистрированные для монокристалла $\mathrm{SrTiO}_{3}$ в его случайной ориентации по отношению к направлению распространения ионных пучков.

потока, превышающий этот параметр в потоках, формируемых обрезающими щелями, на 3-4 порядка. В итоге, применение этих устройств позволило понизить пределы обнаружения примесей в спектрах РФА ПВО еще на два порядка $[10,11]$.

РФА ПВО-измерения могут выполняться либо с использованием специализированных установок [12], либо в рамках малоугловых измерений на стандартных рентгеновских дифрактометрах с включением в измерительную систему энерго-дисперсионного спектрометpa [13]. Поскольку толщина возбуждаемого слоя в РФА ПВО-спектрометрии составляет $3-5 \mathrm{~nm}$, поверхность исследуемого объекта должна быть плоской с низким уровнем волнистости и шероховатости.

\section{3. Особенности ионно-пучковой диагностики материалов}

Важнейшим методом ионно-пучковой диагностики материалов является резерфордовское обратное рассеяние ионных пучков на ядрах атомов, составляющих материальные объекты [14]. Компьютерная аппроксимация экспериментально полученных спектров РОР позволяет получать количественные данные о содержании структурообразующих элементов исследуемого материала.
Обычно для РОР диагностики материалов используются пучки ионов гелия и водорода с энергией $1-3 \mathrm{MeV}$. Поскольку длительное воздействие ионных пучков приводит к появлению структурных искажений и даже аморфизации структуры материалов, аналитическое применение ионно-пучковой диагностики ограничено малыми дозовыми нагрузками, обычно не превышающими $(3-5) \cdot 10^{14} \mathrm{ion} / \mathrm{cm}^{2}$. В отличии от РФА ПВО-измерений, РОР исследования, как и рентгенофлуоресцентный анализ при ионном возбуждении, выполняются в вакуумных камерах. Нормирование зарегистрированных спектров осуществляется на основании учета числа ионов, рассеянных за период измерительной сессии.

На рис. 2 приведены спектры РОР потоков ионов $\mathrm{He}^{+}$и $\mathrm{H}^{+}$, полученные для монокристалла $\mathrm{SrTiO}_{3}$ в положении его случайно ориентации относительно направления распространения ионных потоков. Аппроксимация спектров, выполненная с помощью компьютерной программы RUMPP (модернизированная версии программы RUMP [15]), показала стехиометричность исследованного объекта. При этом аппроксимация спектра РОР потока ионов водорода потребовала проведения дополнительных измерений ввиду наличия нерезерфордовского вклада в рассеяние этих ионов на ядрах атомов кислорода [16]. Концентрация структурных примесей в исследованном монокристаллическом образце опре- 

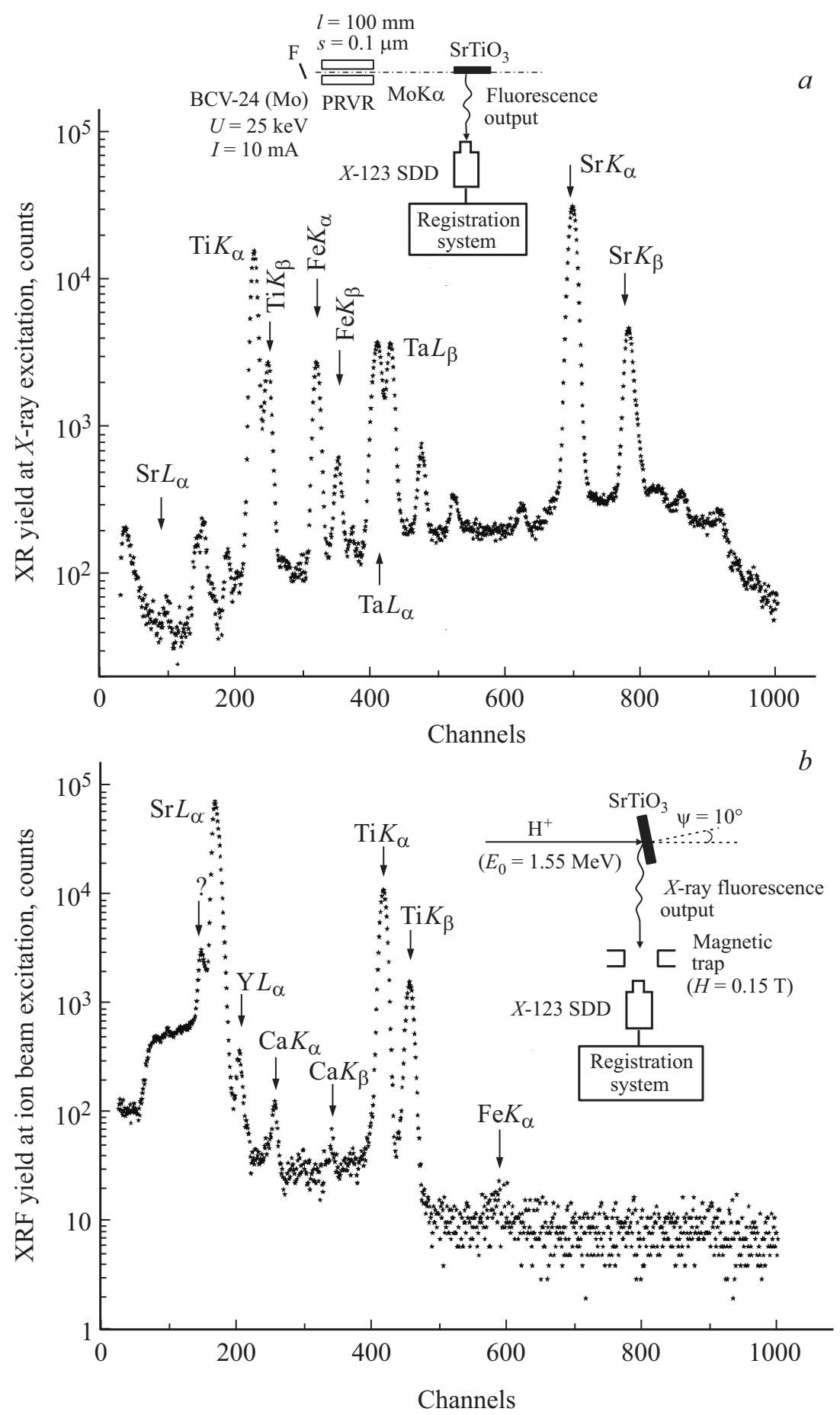

Рис. 3. Спектры РФА ПВО $(a)$ и $\mathrm{PIXE}(b)$, полученные для монокристалла $\mathrm{SrTiO}_{3}$. Энергетическая цена канала для РФА ПВО-измерений $-20 \mathrm{eV} /$ channel, для PIXE $-10 \mathrm{eV} /$ channel.

делялась на основании данных, полученных методами РФА ПВО и РФА при ионном возбуждении. Спектры, зарегистрированные этими методами, показаны на рис. 3. Они показывают, что основными примесями в данном образце являются $\mathrm{Fe}$ и Ta.

При сопоставлении рентгенофлуоресцентных спектров, представленных на рис. 3, обращает на себя внимание, что спектр, зарегистрированный в геомет- рии РФА ПВО измерений, демонстрирует высокую интенсивность для линий $\operatorname{Sr} K_{\alpha}(E=14.164 \mathrm{keV})$ и крайне низкий выход для линии $\operatorname{Sr} L_{\alpha}(E=1.806 \mathrm{keV})$. В то же время, спектр РФА, зарегистрированный в условиях возбуждения пучком протонов с энергией $E=1.07 \mathrm{MeV}$, демонстрирует крайне высокую интенсивность для линии $\operatorname{Sr} L_{\alpha}$ при отсутствии регистрируемой интенсивности для линии $\operatorname{Sr} K_{\alpha}$ (на рис. $3, b$ 

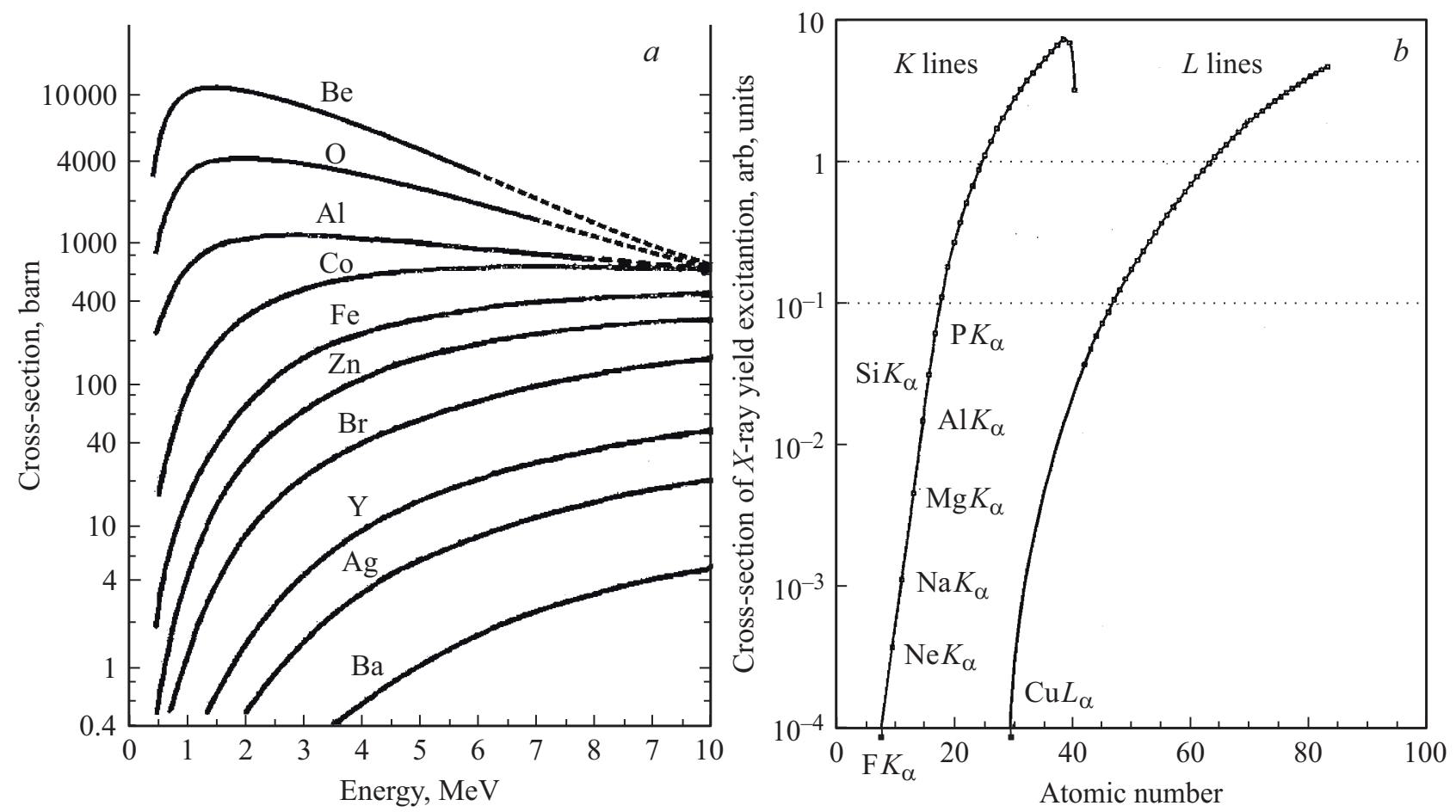

Рис. 4. Сечения возбуждения рентгеновской флуоресценции протонными пучками разных энергий $[18]$ (a) и потоком молибденового излучения, сформированного ПРВР с шириной щели $100 \mathrm{~nm}$ при использовании источника излучения БСВ-27 (Мо) в режиме $U=25 \mathrm{keV}, I=10 \mathrm{~mA}(b)$.

соответствующий энергетический интервал не показан). Интенсивность линии Ті $K_{\alpha}(E=4.51 \mathrm{keV})$ на обоих спектрах показывает промежуточное значение. В целом сравнение этих спектров подтверждает тот факт, что использование электронного, рентгеновского и гамма возбуждения характеристической рентгенофлуоресценции более эффективно для поиска в спектрах выхода высокоэнергетических линий [17], в то время, как линии, соответствующие низкоэнергетическим атомным излучениям, более эффективно возбуждаются ионными пучками [18]. Сравнительные данные по сечениям возбуждения выхода рентгенофлуоресценции пучком протонов и потоком излучения Мо $K_{\alpha}$, сформированным плоским рентгеновским волноводом-резонатором, представлены на рис. 4 [19]. Сравнение представленных зависимостей показывает, что рентгенофлуоресцентные аналитические методы, реализуемые в условиях полного внешнего отражения и ионно-пучкового возбуждения, являются не конкурирующими подходами, а составляют прекрасное дополнение друг другу. Однако, при этом не следует забывать, что эти методы характеризуют возбуждение пространственно не совпадающих объемов изучаемого объекта. РФА ПВО измерения позволяют оценить элементный состав поверхностного слоя этого объекта толщиной 3-5nm, усредненный по площади всей его поверхности. В то же время, в условиях ионно-пучкового возбуждения рентгенофлуоресценции выход характеристических линий формируется в цилиндрическом объеме материала, определяемого сече- нием ионного пучка (обычно диаметром около $1 \mathrm{~mm}$ ) и глубиной его проникновения в материал (от 2 до $20 \mu \mathrm{m}$, в зависимости от типа и энергии ионов в пучке и плотности диагностируемого материала). При этом метод ионно-пучкового возбуждения не лишен влияния матричного фактора, а также зависимости интенсивности регистрируемых рентгенофлуоресцентных линий от коэффициента их поглощения в материале. Вследствие этих факторов метод может считаться лишь полуколичественным. В тот же время по чувствительности в энергетическом интервале $0.5-5.0 \mathrm{keV}$ он значительно превосходит возможности РФА ПВО, а при использовании рентгеновских волноводно-резонансных конструкций способен по чувствительности конкурировать с методами масс-спектрометрической элементной диагностики [20]. Это хорошо иллюстрируется экспериментальными данными, представленными на рис. 5. Он иллюстрирует сравнительную характеристику рентгенофлуоресцентных спектров, полученных обсуждавшимися методами, которые отражают содержание примесных

Содержание примесных элементов в образце тяжелой нефти относительно концентрации атомов серы в ней, полученные на основании данных РФА-исследований

\begin{tabular}{c|c|c|c|c|c|c|c}
\hline $\mathrm{S}$ & $\mathrm{Na}$ & $\mathrm{Cl}$ & $\mathrm{Ca}$ & $\mathrm{V}$ & $\mathrm{Fe}$ & $\mathrm{Ni}$ & $\mathrm{La}$ \\
\hline 1 & 0.005 & 0.005 & 0.005 & 0.015 & 0.001 & 0.005 & 0.0005
\end{tabular}



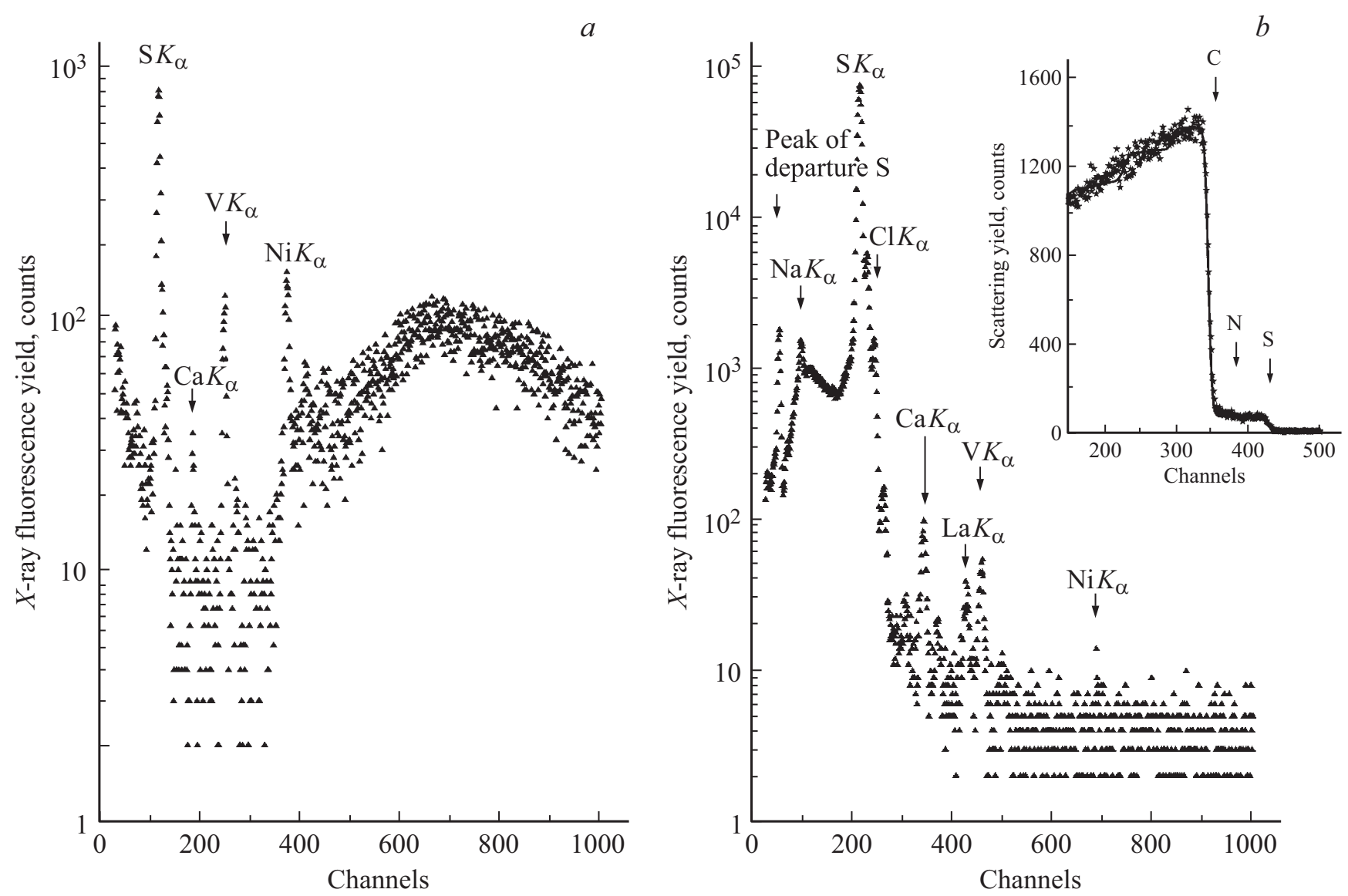

Рис. 5. Спектры PIXЕ $(a)$ и РФА ПВО $(b)$, полученные для пленки тяжелой нефти, нанесенной на Ве подложку. Энергетическая цена каналов для спектра $(a)-10 \mathrm{eV} /$ channel, для спектра $(b)-20 \mathrm{eV} /$ channel. На врезке показан спектр РОР исследованного образца.

элементов в образце тяжелой нефти, пленка которой была высажена на Ве подложке. На врезке показан спектр РОР пучка ионов водорода для этого пленочного образца, на основании которого был определен макросостав нефти $\mathrm{H}_{0.1} \mathrm{C}_{0.87} \mathrm{~S}_{0.028} \mathrm{~N}_{0.002}$. Содержание водорода в образце было определено методом ядер отдачи [21]. В таблице представлен элементный состав содержания примесей в образце нефти относительно содержания в ней атомов серы. Интересным результатом выполненных измерений является диагностика наличия в нефтяном сырье индустриально выделяемого количества ванадия и принципиально диагностируемого наличия атомов лантана. Полученные данные дали основание для комплексной элементной диагностики продуктов всех стадий нефтяного крекинга для выявления процессов концентрирования редких и редкоземельных элементов.

Комплекс ионно-пучковой и рентгенофлуоресцентной диагностики может быть с успехом применен для решения ряда задач медицинской и биологической направленности, а также исследований многослойных тонкопленочных объектов [22], имплантационных [23] и диффузионных структур [24] и изучения дефекта плотности тонкопленочных покрытий.

\section{4. Заключение}

Приведенные в работе экспериментальные данные дают основание ожидать, что совместное использование ионно-пучковой и рентгенофлуоресцентной диагностики материалов является необходимым и достаточным средством для элементной характеризации тонкопленочных покрытий поверхностных слоев изучаемых объектов и пленок сухих остатков жидкостей.

\section{Благодарности}

Авторы благодарят за интерес к работе академика РАН Ю.В. Гуляева.

\section{Финансирование}

Работа выполнена в рамках Государственного задания № 075-00475-19-00 при частичной финансовой поддержке грантов РФФИ № 19-07-00271 и № 18-029-11029.

\section{Конфрликт интересов}

Авторы заявляют, что у них нет конфликта интересов. 


\section{Список литературы}

[1] J.R. Bird, J.S. Williams. Ion beams for material analysis. Academic Press, Sydney. (1989). 719 p.

[2] S.A.E. Johanson, J.L. Campbell, K.G. Molquist. Particle induced $X$-ray emission spectrometry (PIXE). Wiley, N.Y. (1995). $451 \mathrm{p}$

[3] R. Klockenkamper, A. Von Bohlen. Total reflection $X$-ray fluorescence analysis and related methods. 2nd Ed. Wiley, N.Y. (2015). 528 p.

[4] Н.Ф. Лосев. Количественный рентгеноспектральный флуоресцентный анализ. Наука, М. (1969). 336 с.

[5] Quantitative electron-probe microanalysis / Eds V.D. Scott, G. Love. Wiley, N. Y. (1983). 321 p.

[6] E.P. Bertin. Principles and practice of $X$-ray spectrometric analysis. 2nd ed. Plenum press, N.Y. (1975). 1079 p.

[7] R. Jenkins, R.W. Gould, D. Gedcke. Quantitative $X$-ray spectrometry. Dekker, N.Y. (1995). 484 p.

[8] Y. Yoneda, T. Horiuchi. Rev. Sci. Instr. 42, 1069 (1971).

[9] V.K. Egorov, E.V. Egorov. $X$-ray Spectrometry 33, 360 (2004).

[10] В.К. Егоров, Е.В. Егоров, Е.М. Лукьянченко. Наноинженерия 5, 7 (2015).

[11] V.K. Egorov, E.V. Egorov, E.M. Loukianchenko. Aspects Min\&Min. Sci. 2, 1 (2018).

[12] H. Stosnach, A. Gross. S2 Picofox, Rapid and cost-effective monitoring of sludge and wasterwater by TXRF spectrometry. Lab. Report XRF 458. Bruker AXS Inc., Berlin (2015). 4 p.

[13] https:/www.amptek.com/products/sdd-x-ray-detectors-for-xrfeds/x-123sdd- complete-x-ray-spectrometer-with-silicon-driftdetector-sdd

[14] M. Nastasi, J.W. Mayer, Y. Wang. Ion beam analysis, fundaments and application. CRC Press, Boca Raton. (2015). $434 \mathrm{p}$.

[15] L.R. Doolittle. NIM 9, 344 (1985).

[16] E. Rauhala. NIM 12, 447 (1985).

[17] X-ray spectrometry: Recent technological advanced / Eds K. Tsuji, R. Van Grieken. Wiley, Chichester. (2004). 517 p.

[18] T.A. Cahill. Ann. Rev. Nucl. Part. Sci. 30, 211 (1980).

[19] Ion beam for material analysis: conventional and advanced approaches / Eds I. Ahmad, M. Maaza. Intechopen, London. (2018). P. 38.

[20] V.K. Egorov, E.V. Egorov, M.S. Afanas'ev. IOP Conf. Ser.: J. Phys.: Conf. Ser. 1121, 1 (2018).

[21] H. Hofsas. Forward recoil spectroscopy. Plenum, N. Y. (1996). $278 \mathrm{p}$.

[22] Handbook on modern ion beam material analysis. 2nd ed. / Eds Y. Wang, M. Nastasi. Warrendale, Material research society (2009). $441 \mathrm{p}$.

[23] Ion implantation / Eds. by M. Goorsky. Intech, Rijeka. (2012). $436 \mathrm{p}$.

[24] P.L. Clay, E.B. Baxter, D.I. Cherniak. Geochim. Cosmochim. Acta 74, 20, 5906 (2010).

Редактор К.В. Емцев 